\title{
Estudo das crenças de professores e alunos dos cursos de Licenciatura em Letras da UEFS sobre o processo de aprendizagem: oportunizando caminhos de pesquisa
}

\author{
A study of UEFS teachers' and students' beliefs about foreign language \\ learning: providing research paths \\ Milenna Brun ${ }^{1}$ \\ Universidade Estadual de Feira de Santana, Feira de Santana, Bahia, Brasil
}

\begin{abstract}
Resumo: Este artigo apresenta um projeto de pesquisa cujo objetivo é mapear o sistema de crenças de alunos e professores dos cursos de Licenciatura em Letras Vernáculas, Língua Inglesa, Língua Francesa e Língua Espanhola da Universidade Estadual de Feira de Santana acerca (1) do processo de aprendizagem de línguas estrangeiras, (2) dos fatores que influenciam tal processo, e (3) das estratégias eficazes de aprendizagem, através da identificação de semelhanças e diferenças entre estes grupos. Tais crenças impactam e interferem nos resultados obtidos pelos alunos bem como nas estratégias de ensino adotadas pelos professores. A cadeia de influência recíproca é significativa: as crenças dos alunos balizam as estratégias de aprendizagem que eles põem em prática; as crenças dos professores não apenas intervêm naquelas dos alunos, mas, acima de tudo, determinam as formas de organização do ensino e da avaliação. Pretende-se que os resultados da pesquisa permitam compreender as representações de chegada e de saída, os fatores que podem influenciar tais mudanças e, essencialmente, o impacto das crenças na formação dos licenciados.
\end{abstract}

Palavras Chave: Crenças. Aprendizagem de Línguas Estrangeiras. BALLI. Projeto de pesquisa.

\begin{abstract}
This article presents a research project whose objective is to map the belief system of students and teachers of undergraduate courses in Portuguese, English, French and Spanish Languages of Feira de Santana State University about (1) the foreign language learning process, (2) the factors that influence this process, and (3) their effective learning strategies, by identifying their similarities and differences. These beliefs have an impact on and interfere in students results. They also affect teaching strategies. The chain of reciprocal influence is significant. On one hand, students' beliefs guide the learning strategies they put into practice. On the other hand, not only do teachers' beliefs intervene in those of the students, but they also determine teaching and evaluation choices. This research results might allow us to better understand and compare beliefs of freshmen and senior students, the factors that influence probable changes in their beliefs during their educational process and, essentially, the impact of beliefs on teaching training.
\end{abstract}

Keywords: Beliefs. Foreign Language Learning. BALLI. Research Project.

\section{INTRODUÇÃO}

A problemática das crenças e percepções sobre a aprendizagem de línguas estrangeiras tem representado um interesse especial na área de educação para a alteridade e para as línguas-culturas estrangeiras há três décadas. $\mathrm{O}$ inventório BALLI foi desenvolvido de forma pioneira por E. Horwitz, em 1987, e desde então numerosas pesquisas atestam sua validade para assessar crenças de professores e

\footnotetext{
${ }_{1}$ Dra. em Didactologia de Línguas e Culturas Estrangeiras, Université de la Sorbonne Nouvelle, Paris 3, Professora Titular de Língua Inglesa, Departamento de Letras e Artes, Universidade Estadual de Feira de Santana, Bahia, Brasil, milennab@uefs.br
} 
alunos de origens variadas. Nikitina e Furuoka (2006) reexaminaram o instrumento no contexto universitário multicultural e multilinguístico da Malásia e atestaram que o questionário Balli continua sendo adequado para a investigação das crenças sobre aprendizagem de línguas em contextos sociolinguísticos diversificados, independentemente da língua alvo. Estudos representativos demonstraram que, além do capital cultural, as diferenças individuais são fatores que influenciam potencialmente as crenças sobre aprendizagem de línguas estrangeiras.

A pesquisa nessa área também tem indicado que estas crenças são de importância crucial para o sucesso ou o fracasso do processo de aprendizagem (Horwitz, 1999; Yang, 1999, Huang, 2006; Chang \& Shen, 2010; Azar, 2013). No contexto brasileiro, esta problemática também vem sendo analisada por pesquisadores, como demonstra o artigo de Silva (2007) que traça um panorama histórico dos estudos realizados no Brasil. A revisão da literatura do autor sobre o conceito de crenças na área da Linguística Aplicada conclui que existem implicações significativas das crenças tanto no processo de aprendizagem como na formação de professores de línguas. Concordando com Barcelos (2004b), Silva sugere o desenvolvimento de futuras pesquisas sobre crenças, elencando especificamente estudos longitudinais como uma das modalidades pertinentes e necessárias a fim de evidenciar a "(re)construção, evolução e possiveis mudanças das crenças no decorrer dos anos" de formação. As crenças dos professores acerca da aprendizagem de línguas são cruciais nas suas decisões instrucionais e também nas suas práticas educativas (Smith, 1996; Tillema, 2000). Consequentemente, as crenças dos professores podem ter impacto significativo na construção do sistema de crenças dos alunos e no processo de ensino-aprendizagem. Segundo Eric Brun (2015), esta influência no processo educativo cristaliza as representações através da sua transmissão entre as gerações, em um ciclo recursivo infinito. Neste ciclo, os alunos, enquanto receptores, às vezes reproduzem, sem uma reflexão crítica, o que aprenderam com seus professores. A análise do sistema de crenças de professores e alunos do curso de Letras da Uefs pode elucidar elementos que venham apoiar o processo educacional e orientar as ações didáticas a serem implementadas, assim como permitir a desmistificação de idéias preconcebidas e não fundamentadas nas pesquisas recentes nesta área de estudos.

Os cursos de licenciatura em Letras, nas suas modalidades Letras com Espanhol, Letras com Francês, Letras com Inglês e Letras Vernáculas contam com mais de três décadas de implantação e correspondem às expectativas da população oriunda de dezenas de cidades, sobretudo de Feira de Santana e da capital baiana. Tais cursos sofreram diversas reformulações curriculares com vistas ao seu próprio aperfeiçoamento $(1999,2003,2011)$. Um novo currículo e uma nova organização dos Colegiados serão implementados em 2017. Tais iniciativas não são motivadas apenas pela necessidade de adequação à legislação vigente, mas procuram atenuar, se não solucionar, problemas inerentes à formação precária de grande parte dos licenciados em atuação na rede pública de ensino. Ora, a (re) construção de cursos superiores de formação para professores passa pelo efetivo desafio de conhecer quem são os sujeitos principais desse processo. Nesse sentido, estamos iniciando um relevante mapeamento comparativo e longitudinal dessas crenças a fim de 
evidenciar aspectos essenciais que influenciam não apenas o processo de aprendizagem de línguas, mas também as estratégias educativas dos professores.

Tal estudo se justifica também por ser inédito no curso de Letras da Uefs e ser precursor ali de uma linha de pesquisa na área de ensino de línguas. As pesquisas do Departamento de Letras e Artes, inclusive os trabalhos monográficos desenvolvidos pelos alunos na graduação, focam, na sua maioria, em estudos linguísticos e/ou literários retroalimentando as produções dos cursos de pósgraduação stricto sensu oferecidos pelo DLA (Mestrado em Línguística e Mestrado em Literatura e Diversidade Cultural). Tarda a criação de uma linha de pesquisa voltada especificamente para o processo de ensino e aprendizagem de línguas estrangeiras cujos estudos, hoje, são desenvolvidos principalmente pelos Projetos de Extensão (Programa Portal e Programa Palle). Assim, cremos que projetos de pesquisa sobre os aspectos subjetivos do processo de aprendizagem podem contribuir para a formação dos futuros professores de línguas licenciados no DLA, subsidiar a prática docente dos professores-pesquisadores de línguas estrangeiras e fomentar o desenvolvimento da pesquisa discente e docente no âmbito singular da Educação para Línguas e Culturas Estrangeiras, com a possível criação desta Linha de Pesquisa e até mesmo a (re)edição de mais uma turma do Curso de Especialização de Ensino de Língua Inglesa ${ }^{2}$, (pioneiro e único na Bahia; criado e oferecido na Uefs em 2003 e 2004).

\section{PESQUISA SOBRE CRENÇAS NA APRENDIZAGEM DE LÍNGUAS ESTRANGEIRAS: BREVE PERCURSO TEÓRICO}

As crenças são um construto essencial do estudo da aprendizagem e do comportamento humano. No contexto da educação para a alteridade e para as línguas-culturas estrangeiras, elas foram definidas por Victori e Lockhart (1995, p.224) como suposições gerais que o aluno tem sobre si mesmo como aprendiz, sobre fatores que influenciam a aprendizagem e sobre a natureza do processo de aprendizagem.

Segundo Puchata (1999) as crenças são princípios que guiam o comportamento humano. $\mathrm{O}$ autor conceitua as crenças como generalizações de causa e efeito que influenciam nossa representação do mundo.

A definição de crenças parecia muito fluída nos primeiros estudos, mesmo no texto da pioneira Horwitz (1987) que iguala crenças a opiniões ou a idéias preconcebidas. Em 1992, Pajares, após listar uma série de termos considerados sinônimos de crenças nos textos acadêmicos sobre o tema (atitudes, valores, julgamentos, axiomas, opiniões, ideologia, percepções, sistemas conceituais, teorias implícitas, explícitas ou pessoais, perspectivas, entre outros), conclui com a distinção entre crenças e conhecimento. Enquanto as crenças tomam como base a avaliação e o julgamento, o conhecimento fundamenta-se nos fatos objetivos. (p. 313).

2 O Curso de Especialização em Ensino de Língua Inglesa foi concebido em parceria com a Professora Dra. Adelaide Oliveira (UNEB). 
Segundo Peacock (2001) as crenças são compreensões, premissas ou proposições sobre o mundo que são percebidas como verdadeiras. Como advoga Joëlle Aden (2012, p. 270):

Dans les sociétés du XXIe siècle, la didactique des langues ne peut plus se restreindre à un apprentissage des fonctions langagières socialement situées et co-construites, mais elle devrait se poser la question de la place et du rôle de la dimension neuro-psycho-physiologique qui met en jeu notamment, la polyphonie des identités, la multisensorialité dans la perception des discours, l'empathie dans les changements de point de vue, la nature cognitive des émotions, les univers de croyances et les affects des sujets en interaction. ${ }^{1}$

O estudo das crenças fundamenta-se na suposição de que elas têm um papel crítico na definição do comportamento.

A pesquisa de Abdi e Asadi (2015) indica que as crenças dos professores influenciam suas ações na aula, sua atitude e também as crenças dos seus alunos. Por outro lado, as crenças dos alunos afetam sua motivação, atitude, desempenho, proficiência, sucesso, comportamento e uso de estratégias de aprendizagem.

Identificar as crenças dos alunos pode auxiliar na compreensão do possível impacto que elas têm no processo de aprendizagem e de ensino visto que os professores podem usar esse conhecimento para fazer escolhas metodológicas e educativas (Breen, 2001; Bernat \& Gvozdenko, 2005).

Identificar as crenças dos professores também contribui para refletir sobre as discrepâncias entre as perspectivas de professores e alunos e a provável influência que exercem ao longo do curso nas crenças e nos comportamentos dos alunos em classe e face a seu processo de aprendizagem.

O processo de tomada de decisão e o comportamento dos professores assim como suas interações com os alunos modelam seu planejamento e decisões curriculares e determinam, desta maneira, qual conteúdo é ensinado e como ele é ensinado.

Os alunos chegam à sala de aula com idéias preconcebidas sobre a aprendizagem de línguas. Muitos pesquisadores argumentam que existem crenças que são benéficas e podem impulsionar a aprendizagem. Em contrapartida, existem também crenças cujo impacto é bastante negativo. (Horwitz, 1987; White, 1999; Mantle-Bromley, 1995; Mori, 1999).

Barcelos, em 2000, categorizou as pesquisas sobre esta temática em três abordagens distintas organizadas a partir da forma como as crenças são identificadas:

1. A abordagem normativa, que utiliza um conjunto predeterminado de afirmações;

2. A abordagem metacognitiva, que utiliza entrevistas e autorelatórios dos alunos;

\footnotetext{
1 Tradução: "Nas sociedades do século XXI, a didática de línguas não pode se restringir a uma aprendizagem das funções linguísticas socialmente situadas e co-construídas, mas deve questionar o lugar e o papel da dimensão neuro, psíquica e fisiológica que coloca em jogo especificamente a polifonia das identidades, a multisensorialidade na percepção dos discursos, a empatia nas mudanças de perspectivas, a natureza cognitiva das emoções, os universos de crenças e os afetos dos sujeitos em interação".
} 
3. A abordagem contextual, que usa narrativas e metáforas para investigar as crenças.

Embora o uso de questionários estruturados não permita o estudo direto das crenças, que são consideradas como representações relativamente estáveis, ele possibilita a pesquisa abrangendo uma população maior. A vantagem desta abordagem é principalmente a possibilidade de auxiliar os aprendizes a estabalecer objetivos mais realistas, reduzir a ansiedade e desenvolver estratégias de aprendizagem mais eficazes. $\mathrm{Na}$ medida em que as noções preconcebidas são identificadas, elas podem ser retificadas.

O questionário Beliefs About Language Learning Inventory (BALLI) desenvolvido por Elaine Horwitz na Universidade do Texas há três décadas continua sendo o instrumento mais utilizado para investigar crenças de aprendizes a partir de uma abordagem normativa.

O BALLI tem sido bastante usado para identificar as crenças de alunos e sua relação com proficiência, cultura, gênero, escolha de estratégias de aprendizagem e crenças dos professores em diferentes culturas e contextos em diversos países (Ghobadi \& Khodaday no Irã em 2011, Fujiwara na Tailândia em 2011, Zhang \& Cui na China em 2010, Rieger na Hungria em 2009, Bernat \& Lloyd na Austrália em 2007, Nikitina \& Furuoka na Malásia em 2006, Tercanlioglu na Turquia em 2005, Bernat no Vietnam em 2004, Siebert nos Estados Unidos em 2003, Peacock em Hong Kong em 2001, Yang em Taiwan em 1999, Cortazzi \& Jin na China em 1996, Mantle-Bromley nos Estados Unidos em 1995, entre outros).

As pesquisas têm indicado a existência de crenças que podem prejudicar o processo de aprendizagem, a divergência entre as crenças de professores e alunos, o impacto das crenças dos professores nas crenças dos alunos, e também a influência das crenças dos professores nas suas escolhas e ações didáticas.

Peacock (2001) realizou em Hong Kong um dos poucos estudos longitudinais sobre mudanças na crenças de professores em formação. 146 professores de inglês como segunda língua foram acompanhados durante os 3 anos do seu programa de treinamento. Poucas mudanças significativas foram detectadas no final da formação e por isso o pesquisador chegou à conclusão de que era necessário empreender esforços a fim de eliminar crenças prejudiciais antes que os formandos começassem a exercer a profissão de professores de línguas.

Este projeto de pesquisa se esteia em múltiplos estudos realizados em contextos socioculturais diversificados com o uso de um instrumento mundialmente validado e tenta responder a duas inquietações sugeridas por conceituados pesquisadores:

(1) a realização de análises longitudinais no ambiente universitário de formação de professores e;

(2) o cruzamento de dados descritivos sobre crenças de professores e alunos com a efetiva prática educativa. 


\section{MAPEAMENTO DE CRENÇAS SOBRE APRENDIZAGEM DE LÍNGUAS: PROBLEMÁTICA DE PESQUISA}

A pesquisa que será iniciada em 2017.1 sob a supervisão do grupo de pesquisa ELCE (Educação para as Línguas e Culturas Estrangeiras) busca responder, no contexto dos cursos de Letras da UEFS, à seguinte problemática:

1. Quais as crenças de alunos e professores do curso de Letras da Uefs (vernáculas, língua inglesa, língua francesa, língua espanhola) sobre aprendizagem de línguas estrangeiras?

2. Existe uma diferença significativa entre as crenças dos alunos e professores de letras vernáculas e dos alunos e professores de línguas estrangeiras acerca do processo de aprendizagem de línguas?

3. Existe uma diferença significativa entre as crenças dos alunos e professores das diferentes línguas estrangeiras ensinadas na Uefs (inglês, francês e espanhol) em relação ao processo de aprendizagem de línguas?

4. Existe uma diferença significativa entre as crenças dos calouros e dos formandos do curso de Letras na Uefs no que concerne o processo de aprendizagem de línguas? Em caso afirmativo, o que influencia esta mudança?

5. De que maneira as crenças dos professores acerca do processo de ensino e aprendizagem de línguas estrangeiras influenciam aquelas dos alunos?

6. De que maneira as crenças dos professores acerca do processo de ensino e aprendizagem de línguas estrangeiras influenciam e/ou determinam suas escolhas metodológicas e suas práticas didáticas?

\subsection{OBJETIVOS E METAS A SEREM ALCANÇADOS}

O objetivo da pesquisa é mapear de forma comparativa e longitudinal o sistema de crenças de alunos e professores dos cursos de Licenciatura em Letras Vernáculas, Língua Inglesa, Língua Francesa e Língua Espanhola da Uefs acerca do processo de ensino e aprendizagem de línguas estrangeiras.

São objetivos gerais da pesquisa:

1. Contribuir para a formação dos alunos do curso de Licenciatura em Letras - Línguas Estrangeiras - da UEFS;

2. Subsidiar, com base em dados quantitativos e qualitativos, a contínua elaboração dos componentes curriculares e seus respectivos conteúdos programáticos;

3. Fomentar a pesquisa docente e discente na área específica da Educação para Línguas e Culturas Estrangeiras;

Os objetivos específicos do estudo são:

1. Identificar as crenças de professores e alunos dos cursos de Licenciatura em Letras acerca do processo de ensino e aprendizagem de línguas estrangeiras; 
2. Descrever, comparar e analisar as diferenças entre as crenças de professores e alunos de cada curso de Letras (Vernáculas, Inglês, Francês e Espanhol);

3. Contrastar as crenças dos alunos do curso de Letras no início e no final da sua formação;

4. Investigar os fatores que influenciam a modificação das crenças dos alunos no período da sua formação universitária;

5. Examinar de que maneira as crenças dos professores influenciam as crenças dos alunos;

6. Observar e avaliar de que maneira as crenças dos professores influenciam suas práticas didáticas.

\subsection{METODOLOGIA}

Esta pesquisa propõe um levantamento descritivo, comparativo e longitudinal das crenças de professores e alunos dos cursos de Licenciatura em Letras Vernáculas, Língua Inglesa, Língua Francesa e Língua Espanhola da UEFS.

\section{3 ÁREA E POPULAÇÃO:}

O estudo será realizado com todo o universo de professores dos cursos de Letras das áreas de Linguística, Literatura e Estrangeiras (com exceção, portanto, dos professores de Artes e Música) e de alunos (com 18 anos completos) dos quatro cursos ao longo dos nove semestres da formação, acompanhando assim os calouros de 2017.1 até a sua formatura em 2020.1.

\subsection{VARIÁVEIS DA PESQUISA}

- Crenças de professores e alunos acerca do Processo de Ensino e Aprendizagem de Línguas Estrangeiras

\section{Indicadores:}

$\checkmark$ Aptidão para a aprendizagem de línguas estrangeiras

$\checkmark$ Dificuldade no processo de aprendizagem

$\checkmark$ Natureza da aprendizagem de línguas

$\checkmark$ Estratégias de aprendizagem e comunicação

$\checkmark$ Motivação e expectativas

- Escolhas metodológicas e Práticas Didáticas

\section{Indicadores:}

$\checkmark$ Tipo de aulas

$\checkmark$ Língua utilizada

$\checkmark$ Uso de tradução

$\checkmark$ Equilíbrio na exploração das habilidades receptivas e produtivas 


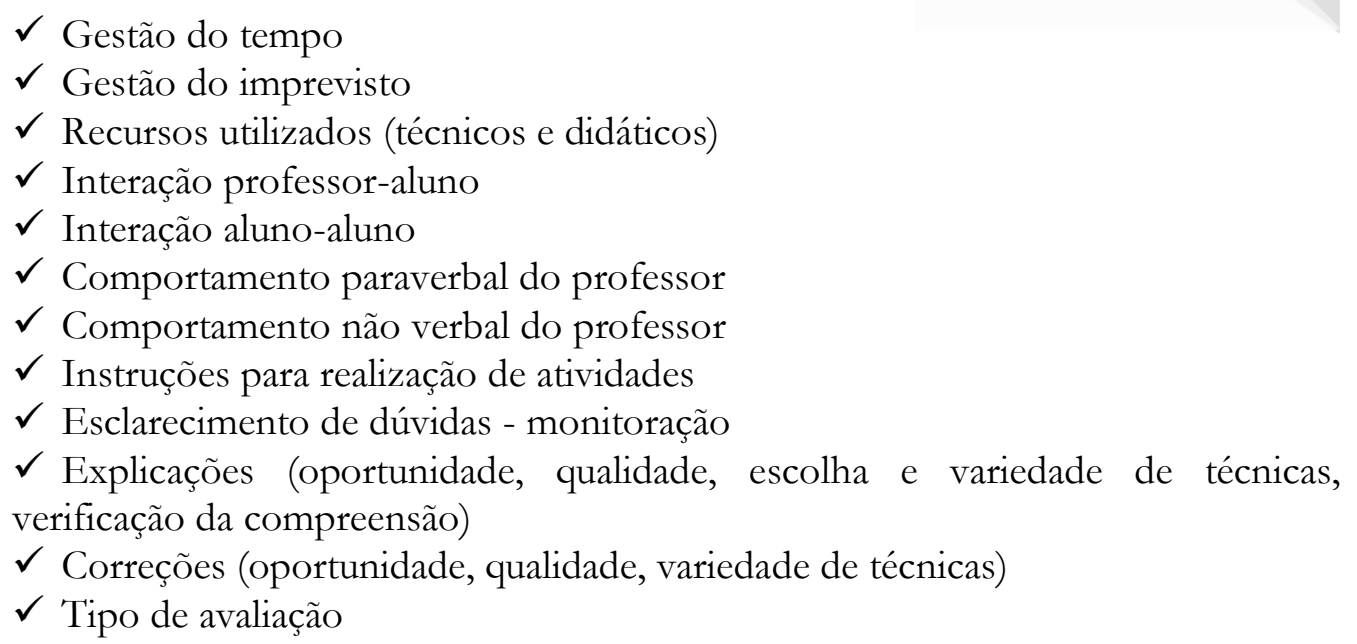

\subsection{ETAPAS E INSTRUMENTOS DE COLETA DE DADOS}

Os dados sobre crenças dos alunos serão investigados através do questionário BALLI versão aluno (Horwitz, 1987) que inclui 34 crenças apresentadas em uma escala Likert. Eles serão coletados em quatro diferentes momentos: no início da formação universitária (calouros), após 1 ano de curso, após 2 anos de curso e no semestre de conclusão.

Os dados sobre crenças dos professores serão investigados através do questionário BALLI versão professor (Horwitz, 1987) em dois momentos: no início da pesquisa (março de 2017 e no semestre que antecede a conclusão da pesquisa (2020.1).

As escolhas metodológicas e as práticas educativas dos professores serão identificadas anualmente através de entrevistas semiestruturadas com professores e alunos.

\subsubsection{Análise de Dados}

Os dados serão analisados estatisticamente e comparados nas seguintes categorias:

Crenças de Alunos Línguas Estrangeiras X Crenças de Alunos de Vernáculas

Crenças de Alunos Línguas Estrangeiras X Crenças de Professores Línguas Estrangeiras

Crenças de Alunos dos Cursos de Letras X Crenças de Professores dos Cursos de Letras

Crenças de Alunos Línguas Estrangeiras Calouros X Crenças de Alunos Línguas Estrangeiras Concluintes

Crenças de Professores X Escolhas Metodológicas e Práticas Educativas

As comparações serão realizadas para o grupo de línguas estrangeiras e também especificamente para cada língua ensinada (alunos de espanhol, de francês e de inglês X professores de espanhol, de francês e de inglês). 


\section{CONCLUSÃO}

A coleta e análise das crenças dos alunos e professores poderá evidenciar possibilidades de estruturar a ação educacional que considere as expectativas dos alunos favorecendo o processo de ensino e aprendizagem. Assim, os resultados esperados do projeto são:

1. Implementar técnicas de intervenção que minimizem as discrepâncias entre as perspectivas de professores e alunos;

2. Contribuir para a formação dos futuros professores de línguas para que possam atuar de forma ativa na compreensão das crenças dos seus próprios alunos;

3. Subsidiar a elaboração de ações didáticas que minimizem o impacto negativo de crenças infundadas dos alunos de línguas estrangeiras;

4. Apoiar as novas disciplinas do eixo de práticas pedagógicas propostas no novo currículo das Licenciaturas de Letras com Línguas Estrangeiras incluindo esta temática e os resultados da pesquisa na formação docente;

5. Criar uma base de dados sobre crenças de professores e alunos sobre processos de aprendizagem na universidade (que pode vir a ser ampliada para outras licenciaturas) evidenciando como as suposições sobre ensino e aprendizagem de línguas estrangeiras são construídas, disseminadas e podem influenciar positiva ou negativamente neste processo;

6. Criar uma linha de pesquisa na Área de Educação para Línguas e Culturas Estrangeiras.

Os impactos previstos com o desenvolvimento da pesquisa são de três ordens: científica, educacional e metodológica.

A criação de uma linha de pesquisa na Área de Educação para Línguas e Culturas Estrangeiras articulada (a) com os Cursos de Pós-graduação oferecidos pelo DLA e (b) com os Programas de Extensão na Área de Ensino e Aprendizagem de Línguas - Programa Portal e Programa Palle - cujas contribuições têm sido comprovadas há décadas tanto para a formação dos alunos de Letras quanto para a comunidade externa, representará um impacto científico considerável.

Os temas a serem desenvolvidos através dos objetivos específicos listados no projeto serão conduzidos por alunos bolsistas e voluntários, podendo ser objeto das Pesquisas que culminam no TCC dos alunos de graduação.

Embora estejamos afastados de uma concepção idealista da relação entre pesquisa $\mathrm{X}$ políticas $\mathrm{X}$ ações educacionais, acreditamos que existe, inegavelmente, ao menos uma porosidade entre o que se produz na pesquisa e o que se realiza nos sistemas educacionais. Os caminhos deste processo de mediação são complexos e, por isso, os benefícios desta pesquisa poderão ser evidenciados apenas a longo prazo, quando alunos e professores da Área de Letras, especificamente da subárea de Estrangeiras, tendo acesso aos resultados, poderão refletir sobre sua prática de ensino e aprendizagem, avaliando de maneira criteriosa as suas escolhas estratégicas didáticas e, consequentemente, aperfeiçoando suas práticas. 


\section{REFERÊNCIAS}

ABDI, Heidar; ASADI, Bahareh. A Synopsis of Researches on Teachers' and Students' Beliefs about Language Learning. International Journal on Studies in English Language and Literature (IJSELL) Vol 3, Issue 4, p. 104-114, 2015.

ADEN, Joelle. La médiation linguistique au fondement du sens partagé : vers un paradigme de l'énaction en didactique des langues, ELA. Études de linguistique appliquée, n¹67, p. 267-284, 2012.

AZAR, Fereshteh Khaffafi; SAEIDI, Mahnaz, The Relationship between Iranian EFL Learners' Beliefs about Language Learning and Their Use of Learning Strategies, English Language Teaching; Vol. 6, N. 11; 2013.

BARCELOS, A. F. Understanding teachers' and students' language learning beliefs in Experience: A Dewyan approach. Tese de Doutorado, Universidade do Alabama. tede.ibict.br/tde/tde_arquivos/1/TDE...01.../AnaMariaAFBarcelos.pdf, 2000.

BARCELOS, A. F. Ser professor de Inglês: crenças, expectativas e dificuldades dos alunos de Letras in: ABRAHÃO. Prática de ensino de língua estrangeira: experiências e reflexões. Campinas, SP: Pontes Editora, 2004b.

BERNAT, E. Investigating Vietnamese ESL learners' beliefs about language learning. English Australia Journal, 21 (2), pp. 40-54, 2004.

BERNAT, E., \& GVOZDENKO, M., Beliefs about language learning: Current knowledge, pedagogical implications and new research directions. TESLJ-EJ, 9(1), 2005.

BERNAT E., \& LLOYD, M., Exploring the gender effect on EFL learners' beliefs about language learning. Australian Journal of Educational and Developmental Psychology, 7, p. 79-91, 2007.

BREEN, M. P. (Ed.), Learner contributions to language learning: New directions in research. Addison-Wesley Longman Limited, 2001.

BRUN, Eric Charles. Sobre a evolução alteritária-etnocêntrica da pré-escola à alfabetização a partir de uma abordagem das línguas-culturas do mundo: uma análise comparativa longitudinal através da expressão oral. Tese de Doutorado, Université d'Aix-Marseille, 2015.

CHANG, C. Y. \& SHEN, M.C., The effects of beliefs about language learning and learning strategy use of junior high school EFL learners in remote districts. Research in Higher Education Journal, 8, 1-8, 2010. 
CORTAZZI, M. \& Jin, L., Cultures of learning: Language classrooms in China. In H. COLEMAN (Ed.), Society and language classroom, p. 169-203, Cambridge: Cambridge University Press, 1996.

FUJIWARA, T., Language Learning Beliefs of thai EFL University Students: Dimensional Structure and Cultural Variations. Electronic Journal of Foreign Language Teaching, 8 (1), pp. 87-107, 2011.

GHOBADI Mohebi, S. \& KHODADADY, E., Investigating university students' beliefs about language learning. RECL Journal, 42 (3), pp. 290-304, 2011.

HORWITZ, E. K., Surveying student beliefs about language learning. In A. WENDEN, Anita; RUBIN, Joan (Eds.). Learner strategies in language learning. Englewood Cliffs, NJ: Prentice-Hall (p.119-129), 1987.

HORWITZ, E.K. Cultural and situational influences on foreign language learners' beliefs about language learning: a review of BALLI studies. System, 27(4), 557-576, 1999.

HUANG, Z.W., Learner beliefs of language learning revisited. Sino-Us English Teaching, 3(3), p. 62-67, 2006.

KUNTZ, Patricia S. Beliefs about Language Learning: The Horwitz Model. (ERIC Document Reproduction Service, No ED397649), 1996 a.

MANTLE-BROMLEY, C., Positive attitudes and realistic beliefs: Links to proficiency. The Modern Language Journal, 79(3), p. 372-386, 1995.

MORI, Y. Epistemological beliefs and language learning beliefs: What do language learners believe about their learning? Language Learning, 49 (3), pp. 377-415, 1999.

NIKITINA, Larisa; FURUOKA, Fumitaka. Re-examining Horwitz's Beliefs About Language Learning Inventory (BALLI) in the Malaysian Context. Eletronic Journal of Foreign Language Teaching, Vol 3, N², pp. 209-219, 2006.

PAJARES, M. F., Teachers' beliefs and educational research: Cleaning up a messy construct. Review of Educational Research, 62 (3), pp. 307-332, 1992.

PEACOCK, M., Pre-service ESL teachers' beliefs about second language learning: A longitudinal study. System, 37, pp. 380-390, 2001.

PUCHATA, H., Beyond materials, techniques and linguistic analysis: the role of motivation, beliefs and identity. Plenary session at the 33rd International IATEFL Annual conference, Edinburg, pp. 64-72, 1999. 
RIEGER, Borbála. Hungarian University Students' Beliefs about language learning: a questionnaire study. WopoLP, vol 3, 2009.

SIEBERT, L. L., Pre-service EFL teachers' beliefs about foreign language learning. The ORTESOL Journal, 21, p. 7-39, 2003.

SILVA, K. A. Crenças sobre o ensino e aprendizagem de línguas na Lingüística Aplicada: um panorama histórico dos estudos realizados no contexto brasileiro. Linguagem \& Ensino (UCPel. Impresso), v. 10, p. 235-271, 2007.

SMITH, D., Teacher decision-making in the adult ESL classroom. In D. FREEMAN; RICHARDS J. (Eds.). Teacher Learning in Language Teaching, p. 197-216. New York: Cambridge University Press, 1996.

TERCANLIOGLU, L., Pre-service EFL teachers' beliefs about foreign language learning and how they relate to gender. Electronic Journal of Research in Educational Psychology, 53 (1), p. 145-162, 2005.

TILLEMA, H. H., Belief change toward self-directed learning in student teachers: Immersion in practice or reflection on action. Teaching and Teacher Education, 16, p. 576-591, 2000.

VICTORI, M.; LOCKHART, W., Enhancing metacognition in self-directed language learning. System, 23(2), p. 223-234, 1995.

WHITE, C., Expectations and emergent beliefs of self-instructed language learners. System, 27 (4), pp. 443-457, 1999.

YANG, N. D., The relationship between learners' beliefs and learning strategy use. System, 27, p. 515-535, 1999.

ZHANG, X.; CUI, G., Learning beliefs of distance foreign language learners in China: A survey study. System, 38, pp. 30-40, 2010. 